\title{
RBF-Based Meshless Method for Large Deflection of Elastic Thin Rectangular Plates with Boundary Conditions Involving Free Edges
}

\author{
Mohammed M. Hussein Al-Tholaia and Husain Jubran Al-Gahtani \\ Civil \& Environmental Engineering Department, King Fahd University of Petroleum \& Minerals (KFUPM), \\ Dhahran 31261, Saudi Arabia \\ Correspondence should be addressed to Husain Jubran Al-Gahtani; hqahtani@kfupm.edu.sa
}

Received 17 February 2016; Revised 22 April 2016; Accepted 12 May 2016

Academic Editor: Evangelos J. Sapountzakis

Copyright (C) 2016 M. M. Hussein Al-Tholaia and H. J. Al-Gahtani. This is an open access article distributed under the Creative Commons Attribution License, which permits unrestricted use, distribution, and reproduction in any medium, provided the original work is properly cited.

\begin{abstract}
An RBF-based meshless method is presented for the analysis of thin plates undergoing large deflection. The method is based on collocation with the multiquadric radial basis function (MQ-RBF). In the proposed method, the resulting coupled nonlinear equations are solved using an incremental-iterative procedure. The accuracy and efficiency of the method are verified through several numerical examples. The inclusion of the free edge boundary condition proves that this method is accurate and efficient in handling such complex boundary value problems.
\end{abstract}

\section{Introduction}

The nonlinear deflection problems arise when using the light weight structures. In several practical light weight structures applications such as fluid tank, aircraft, and hydrospace, flexible plates are commonly used and undergo large deflection. Consequently, the analysis of the plates becomes more difficult. The governing equations become coupled and highly nonlinear [1]. The available analytical methods are based on simplified assumptions and are limited to simple loading and boundary conditions [1-5]. For such complicated problems, numerical methods offer convenient and reliable solutions. The ideal numerical method for the solution of nonlinear partial differential equations (PDEs) such as the one considered here should be high-order accurate, flexible with respect to the geometry, computationally efficient, and easy to implement. The conventional numerical methods that are commonly used usually fulfill one or two of the above criteria, but not all. Finite difference methods (FDM), finite element methods (FEM), and boundary element methods (BEM) have been the dominating methods for the numerical solution of PDEs [6-13]. Referring to the most dominant approach, that is, FEM, it is highly flexible, but it is hard to achieve high-order accuracy and both coding and mesh generation become increasingly difficult as the problem dimension increases. The use of a mesh implies that specific procedures have to be devised just to define the mesh. Also, and to keep the order of the local approximation within reasonable limits, the element size has to be reduced, whenever better approximations are pursued. The extraordinary amount of work, which has been put into FEM research since its early years, has, one way or another, circumvented these and other problems associated with the existence of a mesh and made FEM the dominant approach for most problems in computational mechanics. Accordingly, many sophisticated powerful codes (e.g., ANSYS, ABACUS, and COMSOL) have been established and have proven to be reliable in solving almost any computational mechanics problem. FDM can be made high-order accurate in resolving PDEs but require a structured grid (or a collection of structured grids), which makes it difficult to model features of irregular domain. Furthermore, solutions of PDEs using FDM can be derived from the assumptions of the local interpolation schemes and require a mesh to support the localized approximations; however, the construction of a mesh in two or more dimensions is a nontrivial problem. In 
recent years, BEM has become a powerful alternative to FEM and FDM, especially for problems involving high gradients and stress concentrations. It has been successfully applied to solve the problems of large deflection of thin elastic plates. However, this was possible by devising some techniques to overcome the inherent deficiency of BEM as a self-standing numerical method in handling nonlinearities.

Nevertheless, the possibility of obtaining numerical solutions for PDFs without resorting to element frame has been the goal of many researchers throughout the computational mechanics community for the past three decades or so. One of the earliest meshless methods is the smoothed particle hydrodynamics (SPH) which was developed in 1977 by Lucy et al. $[13,14]$. Since then, many versions and types of the meshless methods have been proposed. Examples are the radial basis function-based collocation method (RBF) by Kansa $[15,16]$, the diffuse element method (DEM) by Nayroles et al. [17], the element-free Galerkin (EFG) method by Belytschko el al. [18], the reproduced kernel particle method (RKPM) by Liu et al. [19], the finite point method by Onate et al. [20], the meshless local Petrov-Galerkin method (MLPG) by Atluri and Zhu [21], and the mesh-free weak-strong form (MWS) by Liu and $\mathrm{Gu}[22]$.

RBF method, as one of the efficient meshless methods, has attracted attention in recent years especially in the area of computational mechanics. In general, RBF method expands the solution of a problem in terms of RBFs and chooses expansion coefficients such that the governing equations and boundary conditions are satisfied at some selected domain and boundary points. This method does not require mesh generation which makes it advantageous for nonlinear problems that require frequent remeshing such as the one considered in this study. The roots of RBF go back to the early 1970s when it was first used for fitting scattered data [23]. In the early 1980s, it was coupled with BEM in a technique called dual reciprocity-boundary element method where the $\mathrm{RBF}$ was employed to transform the domain integrals into boundary integrals [24]. Thereafter, many researchers have used RBF in conjunction with BEM to solve various problems in computational mechanics. The method, however, has not been applied directly to partial differential equations until 1990 by Kansa [15, 16]. Since then, many researchers have suggested several variations to the original method [25-31].

Most of previous applications of meshless methods to large deflection of plates are limited to simple and clamped boundary conditions. The inclusion of free edge boundary condition poses a challenge for both analytical and numerical methods. The difficulty of the free edge boundary condition is due to the enforcement of zero shear on the free boundary of the plate which, for large deflection, involves lengthy and highly nonlinear differential operators as explained in Section 2. In this paper, a multiquadric radial basis function(MQ-RBF-) based meshless model is developed for the solution of large deflection of thin plates with different boundary conditions including free edges. The proposed method has the advantages of simplicity and ease of code implementation. The accuracy of the model is validated through several numerical examples.

\section{Governing Equations}

The governing equilibrium equations of a plate undergoing large deflection can be expressed in terms of the transverse displacement $w$ and a stress function $F$ or in terms of $w$ and the in-plane displacements $u$ and $v$. The summary of both formulations and their corresponding boundary conditions are given below.

2.1. $w$-F Formulation. In this formulation, the governing equations for large elastic deflection of thin plates are represented by two coupled nonlinear differential equations, known as Von Kárámn equations, which are expressed in terms of the displacement $w$ and a stress function $F[1]$ :

$$
\begin{aligned}
& \nabla^{4} w=\frac{q}{D}+\frac{t}{D} \mathrm{NL}(w, F), \\
& \nabla^{4} F=-\frac{E}{2} \mathrm{NL}(w, w),
\end{aligned}
$$

where $q$ is the distributed load, $t$ is the plate thickness, and $D=\left(E t^{3}\right) /\left(12\left(1-v^{2}\right)\right)$ is the flexural rigidity of the plate having elastic constants $E$ and $\nu$, and $\operatorname{NL}(w, F)$ is a nonlinear differential operator given by

$$
\begin{aligned}
& \mathrm{NL}(w, F)=\left[\left(\frac{\partial^{2} F}{\partial y^{2}}\right)\left(\frac{\partial^{2} w}{\partial x^{2}}\right)+\left(\frac{\partial^{2} F}{\partial x^{2}}\right)\left(\frac{\partial^{2} w}{\partial y^{2}}\right)\right. \\
& \left.-2\left(\frac{\partial^{2} F}{\partial x \partial y}\right)\left(\frac{\partial^{2} w}{\partial x \partial y}\right)\right], \\
& \mathrm{NL}(w, w)=\left[2\left(\frac{\partial^{2} w}{\partial x^{2}}\right)-2\left(\frac{\partial^{2} w}{\partial x \partial y}\right)^{2}\right] .
\end{aligned}
$$

The stress function $F$ is related to the membrane forces $N_{x}$, $N_{y}$, and $N_{x y}$ by the following differential operators:

$$
\begin{aligned}
& N_{x}=t\left(\frac{\partial^{2} F}{\partial y^{2}}\right), \\
& N_{y}=t\left(\frac{\partial^{2} F}{\partial x^{2}}\right), \\
& N_{x y}=-t\left(\frac{\partial^{2} F}{\partial x \partial y}\right) .
\end{aligned}
$$

The details for deriving (1) and (2) are given in the classical book by Timoshenko and Woinowsky-Kreiger [1]. The first equation describes the transverse or bending action whereas the second equation represents the in-plane action of the plate. In general, there are two types of in-plane boundary conditions, namely, movable and immovable. The $w-F$ formulation can be utilized directly in the former type where the movable boundary condition is represented by $F=$ $\partial F / \partial n=0$. However, the immovable boundary condition 
$(u=v=0)$ cannot be accurately expressed in terms of the stress function $F$. To overcome this difficulty, (2) can be replaced by two partial differential equations in terms of the in-plane displacements, $u$ and $v$, as described in the following section.

2.2. $u-v-w$ Formulation. Excluding the body forces, the equilibrium equations along $x$ and $y$ are given by

$$
\begin{aligned}
& \frac{\partial N_{x}}{\partial x}+\frac{\partial N_{y x}}{\partial y}=0 \\
& \frac{\partial N_{x y}}{\partial x}+\frac{\partial N_{y}}{\partial y}=0
\end{aligned}
$$

the membrane strains are related to the membrane through the following equations:

$$
\begin{aligned}
\epsilon_{x} & =\frac{N_{x}-v N_{y}}{E t} ; \\
\epsilon_{y} & =\frac{N_{y}-v N_{x}}{E t} ; \\
\gamma_{x y} & =\frac{N_{x y}}{G t} .
\end{aligned}
$$

The membrane strains are also related to the displacements by the following equations:

$$
\begin{aligned}
\epsilon_{x} & =\frac{\partial u}{\partial x}+\frac{1}{2}\left(\frac{\partial w}{\partial x}\right)^{2} \\
\epsilon_{y} & =\frac{\partial v}{\partial y}+\frac{1}{2}\left(\frac{\partial w}{\partial y}\right)^{2} \\
\gamma_{x y} & =\frac{\partial u}{\partial y}+\frac{\partial v}{\partial x}+\frac{\partial w}{\partial x} \frac{\partial w}{\partial y}
\end{aligned}
$$

Using (7) into (6) and then in (5), we get

$$
\begin{aligned}
& \frac{E t}{\left(1-v^{2}\right)}\left\{\frac{\partial^{2} u}{\partial x^{2}}+\frac{\partial w}{\partial x} \frac{\partial^{2} w}{\partial x^{2}}+v\left(\frac{\partial^{2} v}{\partial x \partial y}+\frac{\partial w}{\partial y} \frac{\partial^{2} w}{\partial x \partial y}\right)\right. \\
& \left.+\frac{(1-v)}{2}\left(\frac{\partial^{2} u}{\partial y^{2}}+\frac{\partial^{2} v}{\partial x \partial y}+\frac{\partial w}{\partial y} \frac{\partial^{2} w}{\partial x \partial y}+\frac{\partial w}{\partial x} \frac{\partial^{2} w}{\partial y^{2}}\right)\right\} \\
& =0, \\
& \frac{E t}{\left(1-v^{2}\right)}\left\{\frac{\partial^{2} v}{\partial y^{2}}+\frac{\partial w}{\partial y} \frac{\partial^{2} w}{\partial y^{2}}+v\left(\frac{\partial^{2} u}{\partial x \partial y}+\frac{\partial w}{\partial x} \frac{\partial^{2} w}{\partial x \partial y}\right)\right. \\
& \left.+\frac{(1-v)}{2}\left(\frac{\partial^{2} v}{\partial x^{2}}+\frac{\partial^{2} u}{\partial x \partial y}+\frac{\partial w}{\partial x} \frac{\partial^{2} w}{\partial x \partial y}+\frac{\partial w}{\partial y} \frac{\partial^{2} w}{\partial x^{2}}\right)\right\} \\
& =0 .
\end{aligned}
$$

To complete the $u-v-w$ formulation, (1) is rewritten after replacing the stress function $F$ by the proper terms involving the displacements $u, v$, and $w$. Equation (1) becomes

$$
\begin{aligned}
& D \nabla^{4} w=q+\frac{E t}{\left(1-v^{2}\right)}\left\{\frac { \partial ^ { 2 } w } { \partial x ^ { 2 } } \left[\left(\frac{\partial u}{\partial x}+\frac{1}{2} \frac{\partial w}{\partial x} \frac{\partial w}{\partial x}\right)\right.\right. \\
& \left.+v\left(\frac{\partial v}{\partial y}+\frac{1}{2} \frac{\partial w}{\partial y} \frac{\partial w}{\partial y}\right)\right]+\frac{\partial^{2} w}{\partial y^{2}}\left[\left(\frac{\partial v}{\partial y}+\frac{1}{2} \frac{\partial w}{\partial y} \frac{\partial w}{\partial y}\right)\right. \\
& \left.+v\left(\frac{\partial u}{\partial x}+\frac{1}{2} \frac{\partial w}{\partial x} \frac{\partial w}{\partial x}\right)\right]+(1 \\
& \left.-v) \frac{\partial^{2} w}{\partial x \partial y}\left(\frac{\partial u}{\partial y}+\frac{\partial v}{\partial x}+\frac{\partial w}{\partial x} \frac{\partial w}{\partial y}\right)\right\} .
\end{aligned}
$$

Equations (8) and (9) can be expressed in compact forms as follows:

$$
\begin{aligned}
L_{11}(u)+L_{12}(v) & =\mathrm{NL}_{1}(w) \\
L_{21}(u)+L_{22}(v) & =\mathrm{NL}_{2}(w) \\
\nabla^{4} w & =\frac{q}{D}+\mathrm{NL}_{3}(u, v, w),
\end{aligned}
$$

where

$$
\begin{aligned}
L_{11}= & \frac{2 \partial_{x x}+(1-v) \partial_{y y}}{2\left(1-v^{2}\right)}, \\
L_{12}= & L_{21}=\frac{2 \partial_{x y}}{2(1-v)}, \\
L_{22}= & \frac{2 \partial_{y y}+(1-v) \partial_{x x}}{2\left(1-v^{2}\right)}, \\
\mathrm{NL}_{1}(w) & \\
= & -\frac{(1+v) w_{x y} w_{y}+w_{x}\left(2 w_{x x}+(1-v) w_{y y}\right)}{2\left(1-v^{2}\right)} \\
\mathrm{NL}_{2} & (w) \\
= & -\frac{(1+v) w_{x y} w_{x}+w_{y}\left(2 w_{y y}+(1-v) w_{x x}\right)}{2\left(1-v^{2}\right)} \\
& +\frac{E t w_{y y}}{2 D\left(1-v^{2}\right)}\left(2 v_{y}+w_{y}^{2}+v\left(2 u_{x}+w_{x}^{2}\right)\right) \\
\mathrm{NL}_{3} & (u, v, w) \\
= & \frac{E t w_{x y}}{D(1+v)}\left(u_{y}+v_{x}+w_{x} w_{y}\right) \\
& +\frac{E t w_{x x}}{2 D\left(1-v^{2}\right)}\left(2 u_{x}+w_{x}^{2}+v\left(2 v_{y}+w_{y}^{2}\right)\right)
\end{aligned}
$$

In the above equations, subscript variables denote partial differentiation with respect to the variables. 
2.3. Boundary Conditions. The general boundary conditions for large deflection of plates can be classified into two types.

(1) The first is transverse boundary conditions which are encountered in both small and large deflection formulations. For this type, we will assume that, at each boundary point, there are two prescribed boundary conditions:

(a)

$$
\mathrm{BC}_{w 1}(w)=0
$$

where $\mathrm{BC}_{w 1}(w)= \begin{cases}w, & \text { for simple and clamped edges } \\ V_{n}, & \text { for free edges }\end{cases}$

(b)

$$
\begin{aligned}
& \mathrm{BC}_{w 2}(w)=0, \\
& \text { where } \mathrm{BC}_{w 2}(w)= \begin{cases}\frac{\partial w}{\partial n}, & \text { for a clamped edge } \\
M_{n}, & \text { for simple and free edges, }\end{cases}
\end{aligned}
$$

where $M_{n}$ is the normal bending moment which is given by

$$
\begin{aligned}
& M_{n}=-D\left\{\nu \nabla^{2} w\right. \\
& \left.\quad+(1-\nu)\left(n_{x}^{2} \frac{\partial^{2} w}{\partial x^{2}}+n_{y}^{2} \frac{\partial^{2} w}{\partial y^{2}}+2 n_{x} n_{y} \frac{\partial^{2} w}{\partial x \partial y}\right)\right\} .
\end{aligned}
$$

The shear force $V_{n}$ is composed of a linear and a nonlinear part; that is, $V_{n}=V_{n}^{L}+V_{n}^{\mathrm{NL}}$, where $V_{n}^{L}$ is given by

$$
\begin{aligned}
V_{n}^{L} & =-D\left\{\left(n_{y}\left(1-n_{x}^{2}(v-1)\right)\right) \frac{\partial^{3} w}{\partial y^{3}}\right. \\
& +\left(n_{x}\left(1-n_{y}^{2}(v-1)\right)\right) \frac{\partial^{3} w}{\partial y^{3}} \\
& +n_{x}\left(-2 n_{x}^{2}(v-1)+n_{y}^{2}(v-1)+v\right) \frac{\partial^{3} w}{\partial y^{2} \partial x} \\
& \left.+n_{y}\left(n_{x}^{2}(v-1)-2 n_{y}^{2}(v-1)+v\right) \frac{\partial^{3} w}{\partial x^{2} \partial y}\right\} .
\end{aligned}
$$

The nonlinear part of the shear, $V_{n}^{\mathrm{NL}}$, is given by

$$
\begin{aligned}
V_{n}^{\mathrm{NL}} & =\frac{E t^{2}}{12\left(-1+v^{2}\right)}\left[n_{y} \frac{\partial^{2} F}{\partial x^{2}} \frac{\partial w}{\partial y}-n_{y} \frac{\partial^{2} F}{\partial x \partial y} \frac{\partial w}{\partial x}\right. \\
+ & \left.n_{x} \frac{\partial^{2} F}{\partial y^{2}} \frac{\partial w}{\partial x}-n_{x} \frac{\partial^{2} F}{\partial x \partial y} \frac{\partial w}{\partial y}\right] .
\end{aligned}
$$

The above nonlinear formula for the nonlinear part of the shear force is suitable for the $w-F$ formulation. The nonlinear part of the shear corresponding to the $u-v-w$ formulation can be obtained by replacing the stress function $F$ with the proper derivatives of $u, v$, and $w$. The result is

$$
\begin{aligned}
& V_{n}^{\mathrm{NL}}=\frac{E t}{12(-1+v)}\left[-12 n_{y} \frac{\partial v}{\partial y} \frac{\partial w}{\partial y}-6 n_{y}\left(\frac{\partial w}{\partial x}\right)^{2} \frac{\partial w}{\partial y}\right. \\
& -6 n_{y}\left(\frac{\partial w}{\partial y}\right)^{3}+6 n_{y}(-1+v) \frac{\partial u}{\partial y} \frac{\partial w}{\partial x} \\
& +6 n_{y}(-1+v) \frac{\partial v}{\partial x} \frac{\partial w}{\partial x}-12 n_{y} \nu \frac{\partial u}{\partial x} \frac{\partial w}{\partial y} \\
& -12 n_{x} \frac{\partial u}{\partial x} \frac{\partial w}{\partial x}-6 n_{x}\left(\frac{\partial w}{\partial x}\right)^{3} \\
& +6 n_{x}(-1+v) \frac{\partial u}{\partial y} \frac{\partial w}{\partial y}+6 n_{x}(-1+v) \frac{\partial v}{\partial x} \frac{\partial w}{\partial y} \\
& \left.-6 n_{x}\left(\frac{\partial w}{\partial y}\right)^{2} \frac{\partial w}{\partial x}-12 n_{x} \nu \frac{\partial v}{\partial y} \frac{\partial w}{\partial x}\right],
\end{aligned}
$$

where $n_{x}$ and $n_{y}$ are the $x$ and $y$ components of the unit vector normal to the boundary, respectively.

(2) The second is in-plane boundary conditions which have to be addressed in the case of large deflection formulation. This type of boundary condition is further classified into two types of edge conditions:

(a) Movable edge (free to move in the in-plane directions) which is represented by the following equation:

$$
F=\frac{\partial F}{\partial n}=0
$$

(b) Immovable edge which is represented by

$$
u=v=0 .
$$

In summary, the plate problem with movable edges can be handled by the $w-F$ formulation represented by the governing equations (1) and (2), the lateral boundary conditions (14) and (15), and the in-plane boundary conditions (20), while the plate problem with immovable edges can be handled by the $u-v-w$ formulation represented by the governing equations (8) and (9), the lateral boundary conditions (14) and (15), and the in-plane boundary conditions (21).

\section{RBF Formulation}

Consider the 2D computational domain (Figure 1) that represents the plate geometry. For collocation, we use node points distributed both along the boundary $\left(\underline{x}_{B}^{j}, j=1, \ldots, N_{B}\right)$ and over the interior $\left(\underline{x}_{D}^{j}, j=1, \ldots, N_{D}\right)$. Let $\underline{x}_{p}=\left\{\underline{x}_{B}, \underline{x}_{D}\right\}$, so that the total number of points called poles is $N_{P}=N_{B}+N_{D}$. 


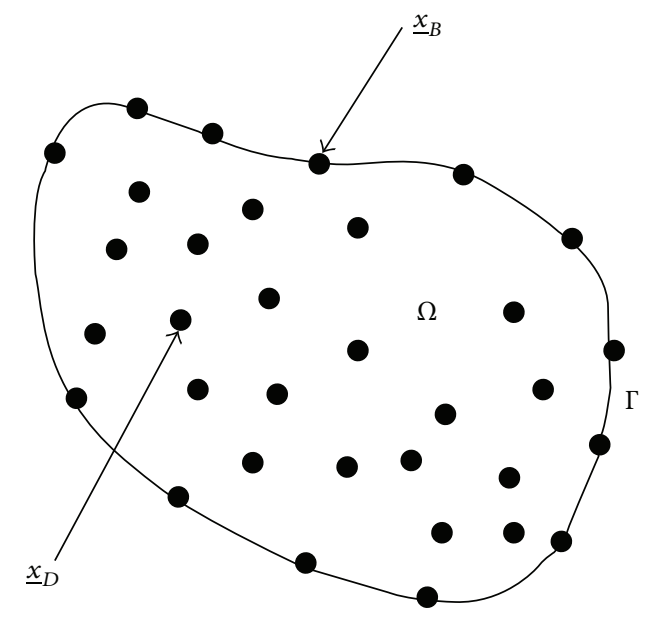

FIGURE 1: Domain and boundary nodes.

The transverse deflections $w$, the stress function $F$, and the in-plane deflections $u$ and $v$ are interpolated linearly by suitable radial basis functions, respectively:

$$
\begin{aligned}
& w(\underline{x})=\sum_{j=1}^{N_{C}} p_{j} w^{*}\left(\left\|\underline{x}-\underline{x}_{C}^{j}\right\|\right) \\
& +\sum_{j=1}^{N_{B}} a_{w}^{j} \mathrm{BC} C_{w 1}\left(\emptyset\left(\left\|\underline{x}-\underline{x}_{B}^{j}\right\|\right)\right) \\
& +\sum_{j=1}^{N_{B}} a_{w}^{j+N_{B}} \mathrm{BC}_{w 2}\left(\emptyset\left(\left\|\underline{x}-\underline{x}_{B}^{j}\right\|\right)\right) \\
& +\sum_{j=1}^{N_{D}} a_{w}^{j+2 N_{B}} \emptyset\left(\left\|\underline{x}-\underline{x}_{D}^{j}\right\|\right), \\
& F(\underline{x})=\sum_{j=1}^{N_{B}} a_{F}^{j} \mathrm{BC} F 1\left(\emptyset\left(\left\|\underline{x}-\underline{x}_{B}^{j}\right\|\right)\right) \\
& +\sum_{j=1}^{N_{B}} a_{F}^{j+N_{B}} \mathrm{BC} C_{F 2}\left(\emptyset\left(\left\|\underline{x}-\underline{x}_{B}^{j}\right\|\right)\right) \\
& +\sum_{j=1}^{N_{D}} a_{F}^{j+2 N_{B}} \emptyset\left(\left\|\underline{x}-\underline{x}_{D}^{j}\right\|\right), \\
& u(\underline{x})=\sum_{j=1}^{N_{P}} a_{v}^{j} \emptyset\left(\left\|\underline{x}-\underline{x}_{P}^{j}\right\|\right), \\
& =\sum_{j=1}^{N_{P}} a_{u}^{j} \emptyset\left(\left\|\underline{x}-\underline{x}_{p}^{j}\right\|\right) ;
\end{aligned}
$$

where

$$
\begin{aligned}
w^{*} & \left(\left\|\underline{x}-\underline{x}_{C}^{j}\right\|\right) \\
& =\left(-\frac{\left(\left\|\underline{x}-\underline{x}_{C}^{j}\right\|\right)^{2}\left(-1+\operatorname{Ln}\left(\left\|\underline{x}-\underline{x}_{C}^{j}\right\|\right)\right)}{8 \pi}\right),
\end{aligned}
$$

$p_{j}$ is the magnitude of the concentrated load acting at the $j$ th point $\left(\underline{x}_{C}^{j}, \underline{y}_{C}^{j}\right)$

$$
\emptyset\left(\left\|\underline{x}-\underline{x}^{j}\right\|\right)=\sqrt{\left(x-x^{j}\right)^{2}+\left(y-y^{j}\right)^{2}+c^{2}},
$$

with a multiquadratic radial basis function (MQ-RBF), $c$ is the shape factor, and $a_{w}^{j}, a_{F}^{j}, a_{u}^{j}$, and $a_{v}^{j}$ are unknown coefficients to be determined by applying the governing equations at the domain points and satisfying the boundary conditions at the boundary points.

For the $w-F$ formulation, the collocation of (1) and (2) yields the following coupled nonlinear algebraic equations:

$$
\begin{aligned}
& {\left[\begin{array}{ccc}
\mathrm{BC}_{w 1}\left(\mathrm{BC}_{w 1}(\emptyset)\right) & \mathrm{BC}_{w 1}\left(\mathrm{BC}_{w 2}(\emptyset)\right) & \mathrm{BC}_{w 1}(\emptyset) \\
\mathrm{BC}_{w 2}\left(\mathrm{BC}_{w 1}(\emptyset)\right) & \mathrm{BC}_{w 2}\left(\mathrm{BC}_{w 2}(\emptyset)\right) & \mathrm{BC}_{w 2}(\emptyset) \\
\left.\nabla^{4} C_{w 1}(\emptyset)\right) & \left(\nabla^{4}\right)\left(\mathrm{BC}_{w 2}(\emptyset)\right) & \nabla^{4}(\emptyset)
\end{array}\right]} \\
& \quad\left\{\begin{array}{c}
a_{w}^{j}, j=1, N_{B} \\
a_{w}^{j+N_{B}}, j=1, N_{B} \\
a_{w}^{j+2 N_{B}}, j=1, N_{D}
\end{array}\right\} \\
& \quad=\left\{\begin{array}{c}
\mathrm{BC}_{w 1}(w)-\mathrm{BC}_{w 1}\left(p_{j} w^{*}\right) \\
\mathrm{BC}_{w 2}(w)-\mathrm{BC}_{w 2}\left(p_{j} w^{*}\right) \\
\frac{q}{D}+\mathrm{NL}(w, F)-\left(\nabla^{4}\right)\left(p_{j} w^{*}\right)
\end{array}\right\},
\end{aligned}
$$$$
\left[\begin{array}{ccc}
\emptyset & \frac{\partial \emptyset}{\partial n} & \emptyset \\
\frac{\partial \emptyset}{\partial n} & \frac{\partial}{\partial n}\left(\frac{\partial \emptyset}{\partial n}\right) & \frac{\partial \emptyset}{\partial n} \\
\nabla^{4}(\emptyset) & \nabla^{4}\left(\frac{\partial \emptyset}{\partial n}\right) & \nabla^{4}(\emptyset)
\end{array}\right]\left\{\begin{array}{c}
a_{F}^{j}, j=1, N_{B} \\
a_{F}^{j+N_{B}}, j=1, N_{B} \\
a_{F}^{j+2 N_{B}}, j=1, N_{D}
\end{array}\right\}
$$$$
=\left\{\begin{array}{c}
0 \\
0 \\
-\frac{E}{2} \mathrm{NL}(w, w)
\end{array}\right\} .
$$ 
TABLE 1: Deflections and stresses for SSSF plate under a uniform load.

\begin{tabular}{lcccccccccccc}
\hline $\bar{q}$ & \multicolumn{3}{c}{$\bar{w}(a / 2, a / 2)$} & \multicolumn{3}{c}{$\bar{w}(a, a / 2)$} & \multicolumn{3}{c}{$\overline{\sigma_{y}}(a / 2, a / 2)$} & \multicolumn{3}{c}{$\overline{\sigma_{y}(a, a / 2)}$} \\
& FEM & RBF & Diff.\% & FEM & RBF & Diff.\% & FEM & RBF & Diff.\% & FEM & RBF & Diff.\% \\
\hline 1.5 & 0.130 & 0.129 & 0.880 & 0.211 & 0.210 & 0.487 & 0.720 & 0.700 & 2.784 & 1.008 & 0.971 & 3.746 \\
3.0 & 0.260 & 0.258 & 0.855 & 0.422 & 0.420 & 0.507 & 1.440 & 1.400 & 2.732 & 2.017 & 1.941 & 3.745 \\
4.5 & 0.390 & 0.387 & 0.852 & 0.633 & 0.630 & 0.558 & 2.156 & 2.099 & 2.672 & 3.026 & 2.914 & 3.703 \\
6.0 & 0.519 & 0.515 & 0.878 & 0.844 & 0.839 & 0.641 & 2.869 & 2.794 & 2.631 & 4.037 & 3.890 & 3.638 \\
7.5 & 0.648 & 0.642 & 0.933 & 1.055 & 1.047 & 0.755 & 3.578 & 3.485 & 2.587 & 5.048 & 4.869 & 3.542 \\
9.0 & 0.777 & 0.769 & 1.016 & 1.267 & 1.255 & 0.896 & 4.281 & 4.170 & 2.611 & 6.060 & 5.850 & 3.462 \\
10.5 & 0.904 & 0.894 & 1.118 & 1.477 & 1.462 & 1.057 & 4.979 & 4.844 & 2.719 & 7.073 & 6.830 & 3.430 \\
12.0 & 1.032 & 1.019 & 1.253 & 1.688 & 1.667 & 1.255 & 5.671 & 5.505 & 2.928 & 8.086 & 7.805 & 3.475 \\
\hline
\end{tabular}

TABLE 2: Deflections and stresses for SSSF plate under a central concentrated load.

\begin{tabular}{ccccccccccccc}
\hline $\bar{p}$ & \multicolumn{3}{c}{$\bar{w}(a / 2, a / 2)$} & \multicolumn{3}{c}{$\bar{w}(a, a / 2)$} & \multicolumn{3}{c}{$\overline{\sigma_{y}}(a / 2, a / 2)$} & \multicolumn{3}{c}{$\overline{\sigma_{y}}(a, a / 2)$} \\
& FEM & RBF & Diff.\% & FEM & RBF & Diff.\% & FEM & RBF & Diff.\% & FEM & RBF & Diff.\% \\
\hline 1 & 0.182 & 0.18 & 1.099 & 0.182 & 0.179 & 1.648 & 3.207 & 3.089 & 3.668 & 0.926 & 0.921 & 0.509 \\
2 & 0.361 & 0.357 & 1.108 & 0.366 & 0.361 & 1.366 & 6.382 & 6.167 & 3.381 & 1.859 & 1.843 & 0.838 \\
3 & 0.536 & 0.537 & 0.187 & 0.551 & 0.534 & 3.085 & 9.502 & 9.188 & 3.304 & 2.805 & 2.761 & 1.582 \\
4 & 0.705 & 0.718 & 1.844 & 0.74 & 0.729 & 1.486 & 12.551 & 12.010 & 4.310 & 3.770 & 3.664 & 2.812 \\
5 & 0.869 & 0.902 & 3.797 & 0.932 & 0.941 & 0.966 & 15.526 & 14.829 & 4.493 & 4.759 & 4.578 & 3.797 \\
6 & 1.027 & 1.063 & 3.505 & 1.128 & 1.173 & 3.989 & 18.430 & 17.581 & 4.611 & 5.775 & 5.501 & 4.748 \\
7 & 1.18 & 1.219 & 3.305 & 1.328 & 1.384 & 4.217 & 21.269 & 20.280 & 4.650 & 6.821 & 6.523 & 4.356 \\
8 & 1.330 & 1.383 & 3.954 & 1.532 & 1.605 & 4.731 & 24.050 & 22.983 & 4.436 & 7.895 & 7.504 & 4.949 \\
\hline
\end{tabular}

TABLE 3: Central and free edge deflections for CCCF plate subjected to a uniform load $\bar{q}$.

\begin{tabular}{lcccccc}
\hline $\bar{q}$ & \multicolumn{3}{c}{$\bar{w}(a / 2, a / 2)$} & \multicolumn{3}{c}{$\bar{w}(a / a / 2)$} \\
& FEM & RBF & Diff.\% & FEM & RBF & Diff.\% \\
\hline 5 & 0.103 & 0.102 & 1.266 & 0.161 & 0.159 & 1.469 \\
10 & 0.207 & 0.204 & 1.225 & 0.323 & 0.318 & 1.508 \\
15 & 0.310 & 0.306 & 1.201 & 0.485 & 0.477 & 1.549 \\
20 & 0.412 & 0.407 & 1.204 & 0.647 & 0.637 & 1.584 \\
25 & 0.514 & 0.508 & 1.234 & 0.810 & 0.797 & 1.606 \\
30 & 0.616 & 0.608 & 1.292 & 0.973 & 0.958 & 1.610 \\
35 & 0.717 & 0.707 & 1.372 & 1.137 & 1.119 & 1.590 \\
40 & 0.817 & 0.805 & 1.471 & 1.302 & 1.282 & 1.543 \\
\hline
\end{tabular}

Similarly, the collocation of (10), (11), and (12), for the $u-v-w$ formulation, yields the following coupled nonlinear algebraic equations:

$$
\begin{aligned}
& {\left[\begin{array}{cccc}
\emptyset & \emptyset & 0 & 0 \\
L_{11}(\emptyset) & L_{11}(\emptyset) & L_{12}(\emptyset) & L_{12}(\emptyset) \\
0 & 0 & \emptyset & \emptyset \\
L_{21}(\emptyset) & L_{21}(\emptyset) & L_{22}(\emptyset) & L_{22}(\emptyset)
\end{array}\right]\left\{\begin{array}{c}
a_{u}^{j}, j=1, N_{B} \\
a_{u}^{j+N_{B}}, j=1, N_{D} \\
a_{v}^{j}, j=1, N_{B} \\
a_{v}^{j+N_{B}}, j=1, N_{D}
\end{array}\right\}} \\
& =\left\{\begin{array}{c}
0 \\
\mathrm{NL}_{1}(w) \\
0 \\
\mathrm{NL}_{2}(w)
\end{array}\right\},
\end{aligned}
$$

$$
\begin{aligned}
& {\left[\begin{array}{cccc}
\mathrm{BC}_{w 1}\left(\mathrm{BC}_{w 1}(\emptyset)\right) & \mathrm{BC}_{w 1}\left(\mathrm{BC}_{w 2}(\emptyset)\right) & \mathrm{BC}_{w 1}(\emptyset) \\
\mathrm{BC}_{w 2}\left(\mathrm{BC}_{w 1}(\emptyset)\right) & \mathrm{BC}_{w 2}\left(\mathrm{BC}_{w 2}(\emptyset)\right) & \mathrm{BC}_{w 2}(\emptyset) \\
\left(\nabla^{4}\right)\left(\mathrm{BC}_{w 1}(\emptyset)\right) & \left(\nabla^{4}\right)\left(\mathrm{BC}_{w 2}(\emptyset)\right) & \nabla^{4}(\emptyset)
\end{array}\right]} \\
& *\left\{\begin{array}{c}
a_{w}^{j}, j=1, N_{B} \\
a_{w}^{j+N_{B}}, j=1, N_{B} \\
a_{w}^{j+2 N_{B}}, j=1, N_{D}
\end{array}\right\} \\
& =\left\{\begin{array}{c}
\mathrm{BC}_{w 1}(w)-\mathrm{BC}_{w 1}\left(p_{j} w^{*}\right) \\
\mathrm{BC}_{w 2}(w)-\mathrm{BC}_{w 2}\left(p_{j} w^{*}\right) \\
\frac{q}{D}+\mathrm{NL}_{3}(u, v, w)-\left(\nabla^{4}\right)\left(p_{j} w^{*}\right)
\end{array}\right\},
\end{aligned}
$$

where the collocations of (10) and (11) have been combined to get (26). For the solution of (25), (26), and (27), the loads are applied incrementally and then solved by iterations until the convergence is satisfied for each incremental load. The nonlinear terms $\left(\mathrm{NL}(w, F), V_{n}^{\mathrm{NL}}\right.$ and $\left.-(E / 2) \mathrm{NL}(w, w)\right)$ in (25) and $\mathrm{NL}_{1}(w), \mathrm{NL}_{2}(w)$, and $\mathrm{NL}_{3}(u, v, w)$, in (26) and (27), are put equal to zero in the first iteration of the first incremental load and then (25), (26), and (27) are solved accordingly to get $w, F, u$, and $v$ functions after the nonlinear terms are evaluated to be used in the next iteration. This procedure is iterated until convergence is satisfied; otherwise, the load increment is reduced. The iterative procedure is repeated by adding load increments and performing iterations until the total load is applied. 
TABLE 4: Central, clamped, and free edge stresses for CCCF plate subjected to a uniform load $\bar{q}$.

\begin{tabular}{lcccccccccccc}
\hline $\bar{q}$ & \multicolumn{3}{c}{$\overline{\sigma_{y}}(a / 2, a / 2)$} & \multicolumn{3}{c}{$\overline{\sigma_{x}}(0, a / 2)$} & \multicolumn{3}{c}{$\overline{\sigma_{y}}(a / 2,0)$} & \multicolumn{3}{c}{$\overline{\sigma_{y}}(a, a / 2)$} \\
& FEM & RBF & Diff.\% & FEM & RBF & Diff.\% & FEM & RBF & Diff.\% & FEM & RBF & Diff.\% \\
\hline 5 & 0.943 & 0.941 & 0.258 & 1.727 & 1.641 & 4.979 & 2.014 & 1.940 & 3.657 & 1.317 & 1.272 & 3.386 \\
10 & 1.884 & 1.880 & 0.178 & 3.452 & 3.281 & 4.967 & 4.026 & 3.880 & 3.631 & 2.635 & 2.545 & 3.429 \\
15 & 2.820 & 2.816 & 0.114 & 5.177 & 4.920 & 4.959 & 6.034 & 5.817 & 3.611 & 3.957 & 3.819 & 3.465 \\
20 & 3.749 & 3.747 & 0.068 & 6.900 & 6.558 & 4.955 & 8.038 & 7.749 & 3.598 & 5.283 & 5.098 & 3.491 \\
25 & 4.671 & 4.669 & 0.041 & 8.620 & 8.193 & 4.956 & 10.037 & 9.676 & 3.592 & 6.614 & 6.383 & 3.500 \\
30 & 5.583 & 5.581 & 0.034 & 10.338 & 9.825 & 4.962 & 12.028 & 11.596 & 3.593 & 7.951 & 7.674 & 3.486 \\
35 & 6.486 & 6.483 & 0.043 & 12.053 & 11.454 & 4.973 & 14.013 & 13.509 & 3.599 & 9.294 & 8.974 & 3.443 \\
40 & 7.378 & 7.373 & 0.064 & 13.767 & 13.080 & 4.986 & 15.991 & 15.414 & 3.609 & 10.642 & 10.283 & 3.368 \\
\hline
\end{tabular}

TABLE 5: Central and free edge deflections for CCCF plate subjected to a central concentrated load $\bar{p}$.

\begin{tabular}{lcccccc}
\hline $\bar{p}$ & \multicolumn{3}{c}{$\bar{w}(a / 2, a / 2)$} & \multicolumn{3}{c}{$\bar{w}(a / a / 2)$} \\
& FEM & RBF & Diff.\% & FEM & RBF & Diff.\% \\
\hline 2 & 0.145 & 0.144 & 0.759 & 0.059 & 0.059 & 0.421 \\
4 & 0.288 & 0.286 & 0.694 & 0.119 & 0.119 & 0.268 \\
6 & 0.425 & 0.429 & 0.941 & 0.181 & 0.179 & 1.137 \\
8 & 0.557 & 0.570 & 2.334 & 0.246 & 0.241 & 2.101 \\
10 & 0.683 & 0.701 & 2.635 & 0.314 & 0.304 & 3.070 \\
12 & 0.802 & 0.831 & 3.616 & 0.385 & 0.369 & 3.989 \\
14 & 0.915 & 0.946 & 3.331 & 0.459 & 0.439 & 4.372 \\
16 & 1.023 & 1.057 & 3.357 & 0.538 & 0.515 & 4.201 \\
\hline
\end{tabular}

\section{Numerical Examples}

In order to examine the effectiveness of the proposed RBF method, the following three examples are considered. In all examples, the loads are assumed to be either uniformly distributed $=q$ or concentrated $=p$, Poisson ratio $\nu$ is assumed 0.3 , and the analysis was performed for several combinations of boundary conditions and shapes. For generality of the solutions, all results are made dimensionless, so that the coordinates, the load, the deflection, and the stress are represented by $\bar{x}=x / a, \bar{y}=y / a, \bar{q}=q a^{4} / E t^{4}, \bar{p}=p a^{2} / E t^{4}$; $\bar{w}=w / t, \bar{\sigma}=\sigma a^{2} / E t^{2}$, respectively. The shape factor, $c$, of the $\mathrm{RBF}$ is changed according to the boundary conditions and the type of load. Its optimum value ranged between 0.3 and 0.8 .

Example 1. Consider a square plate with three simply supported edges and the fourth edge free (denoted by SSSF). All edges are allowed to move in the in-plane directions and hence, the $w-F$ formulation is used. The plate is analyzed for two different load types: a uniformly distributed load $\bar{q}$ ranging from 1.5 to 12 and a central concentrated load $\bar{p}$ ranging from 1 to 8 . The plate is discretized using a uniform node distribution consisting of 36 boundary nodes and 81 domain nodes as shown in Figure 2. The results of the deflections as obtained by RBF and FEM at the center and at the mid-point of the free edge are given in Tables 1 and 2 for the uniform and central concentrated load, respectively. The maximum differences in the deflection at

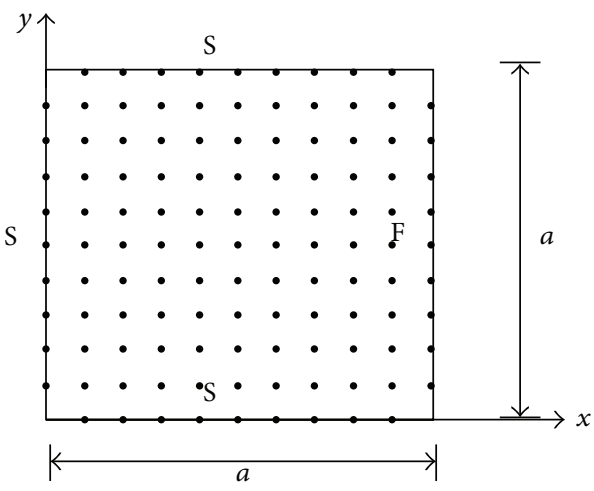

FIGURE 2: Boundary and domain node distribution for the SSSF plate $\left(N_{B}=36 ; N_{D}=81\right)$.

the center of the plate are $1.25 \%$ and $3.95 \%$ for the uniform and concentrated load, respectively, whereas the differences at the free edge of the plate are $1.26 \%$ and $4.73 \%$ for the uniform and concentrated loads, respectively. The maximum differences in the bending stresses at the center of the plate are $2.93 \%$ and $4.49 \%$ for the uniform load and concentrated load, respectively. For the stresses at the mid-point of the free edge, the maximum differences are $3.75 \%$ and $4.95 \%$ for the uniform and concentrated load, respectively.

Example 2. Consider the plate of Example 1 with its simply supported edges replaced by clamped edges and let us denote it by CCCF. The uniform load $\bar{q}$ is incrementally increased from 5 to 40 while the central concentrated load is incrementally increased from 2 to 16 . The plate is discretized using a uniform node distribution similar to the one employed in Example 1. The results of the analysis of this example are given in Tables 3 and 4 for the uniform load and Tables 5 and 6 for the central concentrated load. The results show a good agreement between RBF and FEM solutions for the uniform load with a maximum difference of $1.61 \%$ in the deflection results and a maximum difference of $4.99 \%$ in the stress results. For the concentrated load, the results show a reasonable agreement between the two solutions with a maximum difference of $4.37 \%$ in the deflection results and a maximum difference of $4.88 \%$ in the stress results. 
TABLE 6: Central, clamped, and free edge stresses for CCCF plate subjected to a central concentrated load $\bar{p}$.

\begin{tabular}{lcccccccccccc}
\hline $\bar{p}$ & \multicolumn{3}{c}{$\overline{\sigma_{y}(a / 2, a / 2)}$} & \multicolumn{3}{c}{$\overline{\sigma_{x}}(0, a / 2)$} & \multicolumn{3}{c}{$\overline{\sigma_{y}}(a / 2,0)$} & \multicolumn{3}{c}{$\overline{\sigma_{y}}(a, a / 2)$} \\
& FEM & RBF & Diff.\% & FEM & RBF & Diff.\% & FEM & RBF & Diff.\% & FEM & RBF & Diff.\% \\
\hline 2 & 5.587 & 5.434 & 2.741 & 1.648 & 1.656 & 0.530 & 1.829 & 1.793 & 1.924 & 0.607 & 0.591 & 2.680 \\
4 & 11.117 & 10.696 & 3.794 & 3.275 & 3.313 & 1.158 & 3.631 & 3.569 & 1.709 & 1.221 & 1.183 & 3.128 \\
6 & 16.544 & 15.895 & 3.928 & 4.866 & 4.970 & 2.145 & 5.386 & 5.256 & 2.405 & 1.849 & 1.780 & 3.699 \\
8 & 21.840 & 21.151 & 3.153 & 6.411 & 6.629 & 3.412 & 7.080 & 7.249 & 2.394 & 2.496 & 2.397 & 3.955 \\
10 & 26.992 & 26.107 & 3.276 & 7.905 & 8.231 & 4.125 & 8.708 & 8.975 & 3.064 & 3.167 & 3.037 & 4.112 \\
12 & 31.999 & 31.009 & 3.093 & 9.350 & 9.758 & 4.357 & 10.271 & 10.599 & 3.189 & 3.867 & 3.694 & 4.481 \\
14 & 36.870 & 35.710 & 3.145 & 10.749 & 11.230 & 4.481 & 11.772 & 12.324 & 4.686 & 4.600 & 4.377 & 4.837 \\
16 & 41.614 & 40.216 & 3.361 & 12.105 & 12.682 & 4.764 & 13.217 & 13.834 & 4.672 & 5.368 & 5.106 & 4.881 \\
\hline
\end{tabular}

TABLE 7: Central and free edge deflections for CCCF-IM plate under a uniform load.

\begin{tabular}{|c|c|c|c|c|c|c|}
\hline \multirow{2}{*}{$\bar{q}$} & \multicolumn{3}{|c|}{$\bar{w}(a / 2, a / 2)$} & \multicolumn{3}{|c|}{$\bar{w}(a / a / 2)$} \\
\hline & FEM & $\mathrm{RBF}$ & Diff.\% & FEM & $\mathrm{RBF}$ & Diff. $\%$ \\
\hline 15 & 0.292 & 0.284 & 2.730 & 0.426 & 0.412 & 3.318 \\
\hline 30 & 0.518 & 0.500 & 3.411 & 0.699 & 0.667 & 4.530 \\
\hline 45 & 0.694 & 0.670 & 3.475 & 0.887 & 0.846 & 4.658 \\
\hline 60 & 0.836 & 0.799 & 4.488 & 1.032 & 0.985 & 4.506 \\
\hline 75 & 0.957 & 0.913 & 4.601 & 1.150 & 1.100 & 4.415 \\
\hline
\end{tabular}

TABLE 8: Central, clamped, and free edge stresses for CCCF-IM plate under a uniform load.

\begin{tabular}{ccccccccccccc}
\hline $\bar{q}$ & \multicolumn{3}{c}{$\overline{\sigma_{y}}(a / 2, a / 2)$} & \multicolumn{3}{c}{$\overline{\sigma_{x}}(0, a / 2)$} & \multicolumn{3}{c}{$\overline{\sigma_{y}}(a / 2,0)$} & \multicolumn{3}{c}{$\overline{\sigma_{y}}(a, a / 2)$} \\
& FEM & RBF & Diff.\% & FEM & RBF & Diff.\% & FEM & RBF & Diff.\% & FEM & RBF & Diff.\% \\
\hline 15 & 2.625 & 2.561 & 2.411 & 5.029 & 4.903 & 2.517 & 5.804 & 5.653 & 2.595 & 3.452 & 3.349 & 2.997 \\
30 & 4.579 & 4.424 & 3.394 & 9.517 & 9.160 & 3.756 & 10.815 & 10.425 & 3.604 & 5.637 & 5.440 & 3.498 \\
45 & 6.004 & 5.772 & 3.858 & 13.505 & 12.959 & 4.048 & 15.165 & 14.500 & 4.388 & 7.121 & 6.860 & 3.654 \\
60 & 7.088 & 6.815 & 3.849 & 17.122 & 16.378 & 4.347 & 19.058 & 18.164 & 4.691 & 8.247 & 7.943 & 3.692 \\
75 & 7.949 & 7.610 & 4.260 & 20.460 & 19.557 & 4.414 & 22.622 & 21.509 & 4.921 & 9.168 & 8.816 & 3.837 \\
\hline
\end{tabular}

Example 3. Consider the plate of Example 2 but with immovable edges (CCCF-IM). The solution is obtained using $u-v-w$ formulation. The plate is analyzed under two different load types: uniformly distributed load $\bar{q}$ ranging from 15 to 75 and a central and concentrated load $\bar{p}$ ranging from 4 to 20 . The results (Tables 7-10), once again, show a good agreement between RBF and FEM solutions. For the uniform load, the maximum difference is $4.66 \%$ in the deflection results and $4.92 \%$ in the stress results while, for the concentrated load, the maximum difference is $4.25 \%$ in the deflection and $4.69 \%$ in the stress results.

\section{Conclusions}

An RBF-based meshless method has been presented for the solution of large deflection of thin plates with different boundary conditions including free edges. The method is based on collocation with MQ-RBF. The load types considered in the study are uniform and concentrated. The boundary conditions covered are clamped, simply supported, free, movable, and immovable conditions. The FEM-based software COMSOL has been used to verify the RBF results.
TABLE 9: Central and free edge deflections for CCCF-IM plate under a central concentrated load.

\begin{tabular}{lcccccc}
\hline $\bar{p}$ & \multicolumn{3}{c}{$\bar{w}(a / 2, a / 2)$} & \multicolumn{3}{c}{$\bar{w}(a / a / 2)$} \\
& FEM & RBF & Diff.\% & FEM & RBF & Diff.\% \\
\hline 4 & 0.281 & 0.282 & 0.204 & 0.114 & 0.114 & 0.369 \\
8 & 0.521 & 0.534 & 2.550 & 0.214 & 0.218 & 1.884 \\
12 & 0.717 & 0.739 & 3.062 & 0.297 & 0.304 & 2.334 \\
16 & 0.880 & 0.912 & 3.627 & 0.367 & 0.376 & 2.412 \\
20 & 1.020 & 1.064 & 4.251 & 0.429 & 0.446 & 4.048 \\
\hline
\end{tabular}

The proposed RBF meshless method has the advantage of being simple, easy to implement in a computer code, and reasonably accurate and therefore can qualify as an alternative numerical technique for the solution of highly nonlinear boundary value problems.

\section{Competing Interests}

The authors declare that they have no competing interests. 
TABLE 10: Central, clamped, and free edge stresses for CCCF-IM plate under a central concentrated load.

\begin{tabular}{lcccccccccccc}
\hline $\bar{p}$ & \multicolumn{3}{c}{$\overline{\sigma_{y}(a / 2, a / 2)}$} & \multicolumn{3}{c}{$\overline{\sigma_{x}}(0, a / 2)$} & \multicolumn{3}{c}{$\overline{\sigma_{y}(a / 2,0)}$} & \multicolumn{3}{c}{$\overline{\sigma_{y}}(a, a / 2)$} \\
& FEM & RBF & Diff.\% & FEM & RBF & Diff.\% & FEM & RBF & Diff.\% & FEM & RBF & Diff.\% \\
\hline 4 & 11.034 & 10.873 & 1.460 & 3.210 & 3.160 & 1.559 & 3.560 & 3.467 & 2.602 & 1.165 & 1.149 & 1.454 \\
8 & 21.373 & 21.053 & 1.498 & 6.023 & 5.898 & 2.074 & 6.665 & 6.461 & 3.061 & 2.135 & 2.103 & 1.487 \\
12 & 30.946 & 30.469 & 1.542 & 8.408 & 8.143 & 3.150 & 9.289 & 8.988 & 3.246 & 2.899 & 2.960 & 2.085 \\
16 & 39.913 & 39.171 & 1.860 & 10.470 & 10.121 & 3.334 & 11.556 & 11.165 & 3.386 & 3.516 & 3.599 & 2.364 \\
20 & 48.420 & 47.213 & 2.492 & 12.296 & 11.719 & 4.692 & 13.569 & 14.075 & 3.727 & 4.029 & 4.210 & 4.505 \\
\hline
\end{tabular}

\section{Acknowledgments}

The authors would like to express their appreciation to King Fahd University of Petroleum \& Minerals for supporting this study.

\section{References}

[1] S. P. Timoshenko and S. Woinowsky-Kreiger, Theory of Plates and Shells, McGraw-Hill, New York, NY, USA, 1959.

[2] C. Augural, Stresses in Plates and Shells, McGraw-Hill, New York, NY, USA, 1999.

[3] G. H. Little, "Efficient large deflection analysis of rectangular plates with general transverse form of displacement," Computers and Structures, vol. 71, no. 3, pp. 333-352, 1999.

[4] G. H. Little, "Large deflections of rectangular plates with transverse edges remaining straight," Computers and Structures, vol. 71, no. 3, pp. 353-357, 1999.

[5] L. S. Ramachandra and D. Roy, "A novel technique in the solution of axisymmetric large deflection analysis of a circular plate," Journal of Applied Mechanics, vol. 68, no. 5, pp. 814-816, 2001.

[6] G. Papakaliatakis and T. E. Simos, "A finite difference method for the numerical solution of fourth-order differential equations with engineering applications," Computers \& Structures, vol. 65, no. 4, pp. 491-495, 1997.

[7] M. Duan and M. Mahendran, "Large deflection analyses of skew plates using hybrid/mixed finite element method," Computers and Structures, vol. 81, no. 13, pp. 1415-1424, 2003.

[8] R. Szilard, Theories and Applications of Plate Analysis, John Wiley \& Sons, New Jersey, NJ, USA, 2004.

[9] J. T. Katsikadelis and M. S. Nerantzaki, "Non-linear analysis of plates by the analog equation method," Computational Mechanics, vol. 14, no. 2, pp. 154-164, 1994.

[10] A. El-Zafrany, S. Fadhil, and M. Debbih, "An efficient approach for boundary element bending analysis of thin and thick plates," Computers \& Structures, vol. 56, no. 4, pp. 565-576, 1995.

[11] A. C. J. Luo and J. Mote, "Asymmetric responses of rotating, thin disks experiencing large deflections," Computers \& Mathematics with Applications, vol. 45, no. 1-3, pp. 217-228, 2003.

[12] E. Byklum and J. Amdahl, "A simplified method for elastic large deflection analysis of plates and stiffened panels due to local buckling," Thin-Walled Structures, vol. 40, no. 11, pp. 925-953, 2002.

[13] L. B. Lucy, "A numerical approach to the testing of the fission hypothesis," The Astronomical Journal, vol. 82, pp. 1013-1024, 1977.

[14] R. A. Gingold and J. J. Monaghan, "Smoothed particle hydrodynamics: theory and application to non-spherical stars," Monthly
Notices of the Royal Astronomical Society, vol. 181, no. 3, pp. 375389, 1977.

[15] E. J. Kansa, "Multiquadrics—a scattered data approximation scheme with applications to computational fluid-dynamics. I. Surface approximations and partial derivative estimates," Computers \& Mathematics with Applications, vol. 19, no. 8-9, pp. 127-145, 1990.

[16] E. J. Kansa, "Multiquadrics-a scattered data approximation scheme with applications to computational fluid-dynamics-II solutions to parabolic, hyperbolic and elliptic partial differential equations," Computers \& Mathematics with Applications, vol. 19, no. 8-9, pp. 147-161, 1990.

[17] B. Nayroles, G. Touzot, and P. Villon, "Generalizing the finite element method: diffuse approximation and diffuse elements," Computational Mechanics, vol. 10, no. 5, pp. 307-318, 1992.

[18] T. Belytschko, Y. Y. Lu, and L. Gu, "Element-free Galerkin methods," International Journal for Numerical Methods in Engineering, vol. 37, no. 2, pp. 229-256, 1994.

[19] W. K. Liu, S. Jun, and Y. F. Zhang, "Reproducing kernel particle methods," International Journal for Numerical Methods in Fluids, vol. 20, no. 8-9, pp. 1081-1106, 1995.

[20] E. Onate, S. Idelsohn, O. C. Zienkiewicz, and R. L. Taylor, "A finite point method in computational mechanics. Applications to convective transport and fluid flow," International Journal for Numerical Methods in Engineering, vol. 39, no. 22, pp. 3839$3866,1996$.

[21] S. N. Atluri and T. Zhu, "A new Meshless Local Petrov-Galerkin (MLPG) approach in computational mechanics," Computational Mechanics, vol. 22, no. 2, pp. 117-127, 1998.

[22] G. R. Liu and Y. T. Gu, "A meshfree method: meshfree weakstrong (MWS) form method, for 2-D solids," Computational Mechanics, vol. 33, no. 1, pp. 2-14, 2003.

[23] R. L. Hardy, "Multiquadric equations of topography and other irregular surfaces," Journal of Geophysical Research, vol. 76, no. 8, pp. 1905-1915, 1971.

[24] D. Nardini and C. A. Brebbia, "A new approach to free vibration analysis using boundary elements," Applied Mathematical Modelling, vol. 7, no. 3, pp. 157-162, 1983.

[25] C. M. C. Roque, A. J. M. Ferreira, A. M. A. Neves, C. M. M. Soares, J. N. Reddy, and R. M. N. Jorge, "Transient analysis of composite plates by radial basis functions in a pseudospectral framework," Computers \& Structures, vol. 89, no. 1-2, pp. 161169, 2011.

[26] C. M. C. Roque, D. Cunha, C. Shu, and A. J. M. Ferreira, "A local radial basis functions-finite differences technique for the analysis of composite plates," Engineering Analysis with Boundary Elements, vol. 35, no. 3, pp. 363-374, 2011.

[27] S. Xiang, H. Shi, K.-M. Wang, Y.-T. Ai, and Y.-D. Sha, “Thin plate spline radial basis functions for vibration analysis of clamped 
laminated composite plates," European Journal of MechanicsA/Solids, vol. 29, no. 5, pp. 844-850, 2010.

[28] A. J. M. Ferreira, C. M. C. Roque, A. M. A. Neves, R. M. N. Jorge, C. M. M. Soares, and J. N. Reddy, "Buckling analysis of isotropic and laminated plates by radial basis functions according to a higher-order shear deformation theory," Thin-Walled Structures, vol. 49, no. 7, pp. 804-811, 2011.

[29] H. J. Al-Gahtani and M. Naffa'a, "RBF meshless method for large deflection of thin plates with immovable edges," Engineering Analysis with Boundary Elements, vol. 33, no. 2, pp. 176-183, 2009.

[30] M. Naffa and H. J. Al-Gahtani, "RBF-based meshless method for large deflection of thin plates," Engineering Analysis with Boundary Elements, vol. 31, no. 4, pp. 311-317, 2007.

[31] M. M. Hussein Al-Tholaia and H. Jubran Al-Gahtani, "RBFbased meshless method for large deflection of elastic thin plates on nonlinear foundations," Engineering Analysis with Boundary Elements, vol. 51, pp. 146-155, 2015. 


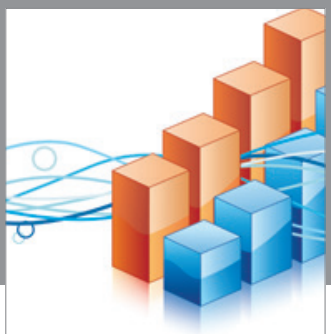

Advances in

Operations Research

vatem alat4

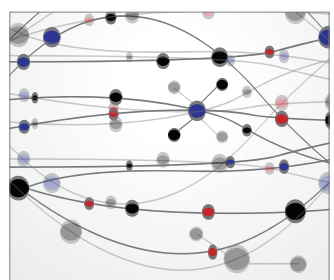

\section{The Scientific} World Journal
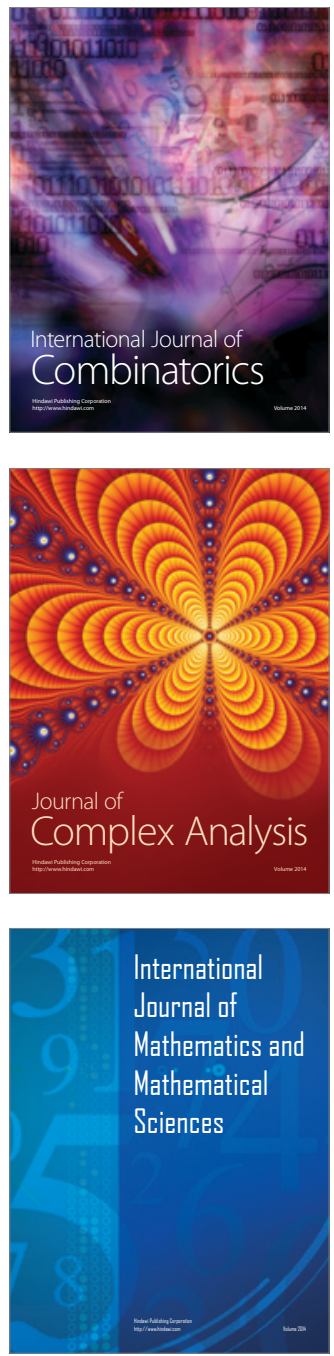
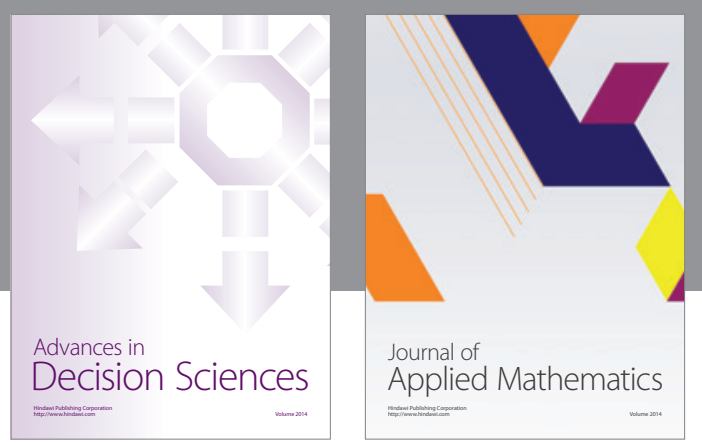

Algebra

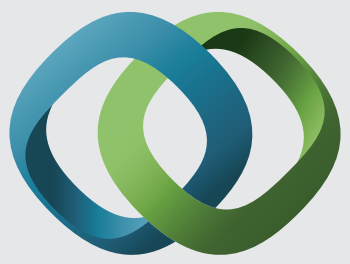

\section{Hindawi}

Submit your manuscripts at

http://www.hindawi.com
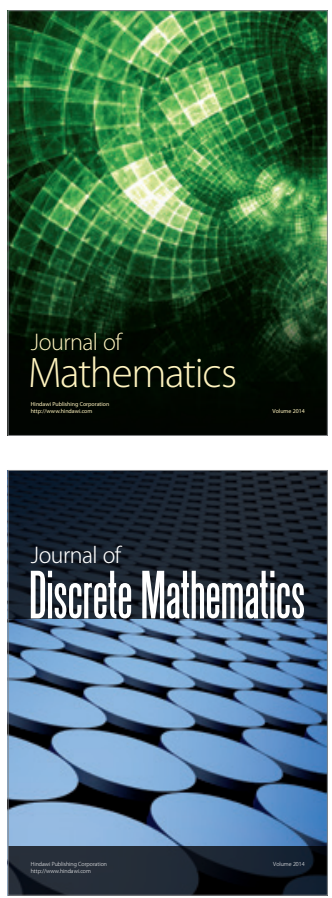

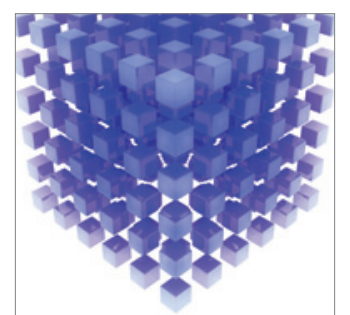

Mathematical Problems in Engineering
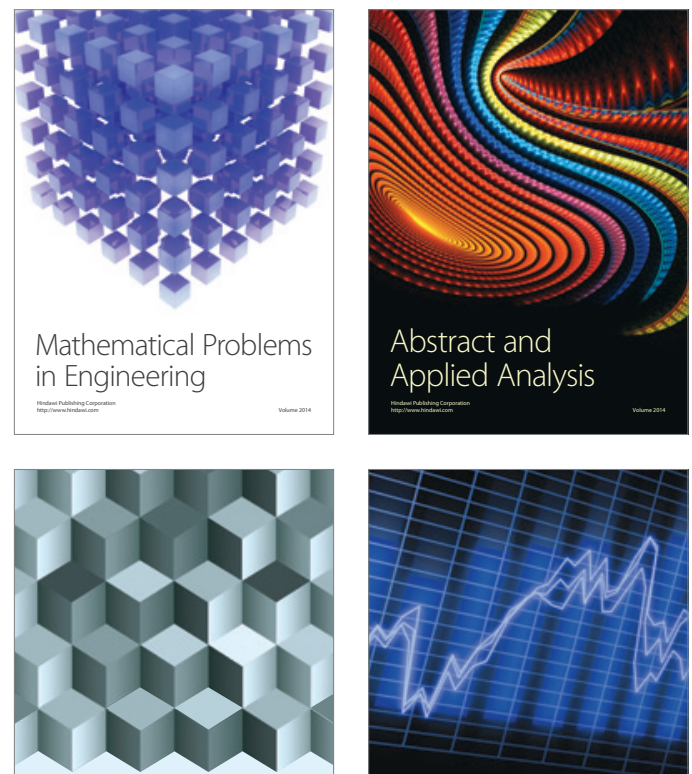

Journal of

Function Spaces

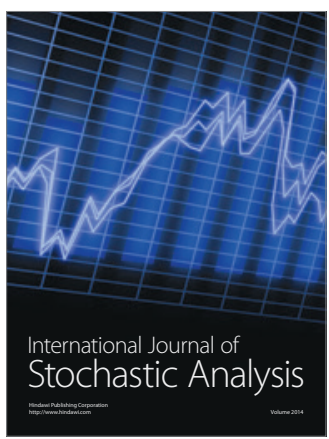

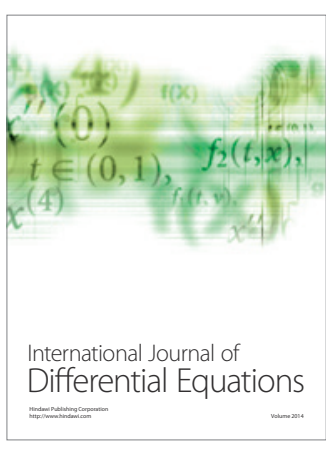
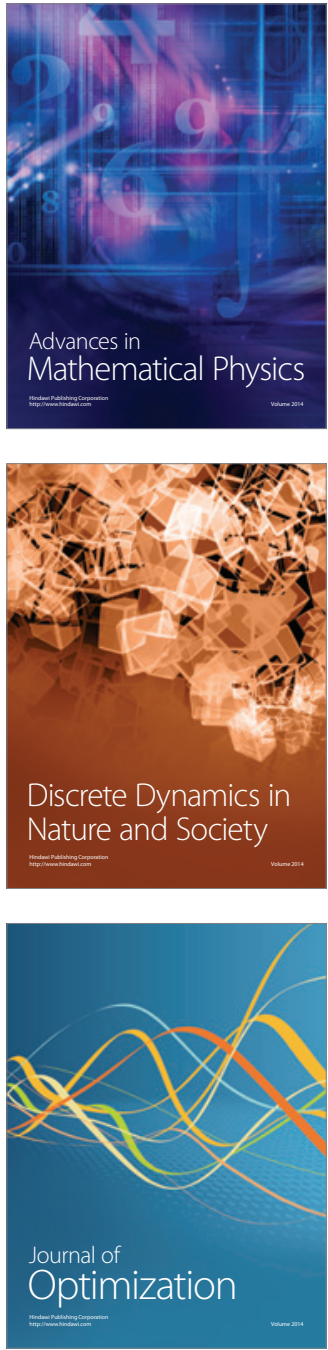